\title{
Chiral Primary Amine/Ketone Cooperative Catalysis for Asymmetric $\alpha$ - Hydroxylation with Hydrogen Peroxide
}

\author{
Mao Cai ${ }^{a b}{ }^{\text {Kaini Xu}},{ }^{c}$ Yuze Li, ${ }^{b d}$ Zongxiu Nie ${ }^{b d}$ Long Zhang ${ }^{* c}$ and Sanzhong Luo*ac \\ ${ }^{a}$ Beijing National Laboratory for Molecular Sciences, Key Laboratory of Molecular Recognition and \\ Function, Institute of Chemistry, Chinese Academy of Sciences, Beijing 100190, China \\ ${ }^{b}$ School of Chemical Science, University of Chinese Academy of Sciences, Beijing 100049, China \\ ${ }^{c}$ Center of Basic Molecular Science, Department of Chemistry, Tsinghua University, Beijing, 100084, China \\ ${ }^{d}$ Beijing National Laboratory for Molecular Sciences, Key Laboratory for Analytical Chemistry for Living \\ Biosystems, Institute of Chemistry, the Chinese Academy of Sciences, Beijing 100190, China
}

\begin{abstract}
Carbonyl and amine are yin and yang in organocatalysis that mutually activate and transform each other. As intrinsically reacting partners, carbonyl and amine tend to condensate, depleting their individual activity when employed as catalysts. Though widely established as prominent catalytic strategies, aminocatalysis and carbonyl catalysis seems not coexist well and a cooperative amine/carbonyl dual catalysis remains virtually unknown. Here we report a cooperative primary amine and ketone dual catalysis in the asymmetric $\alpha$ hydroxylation with $\mathrm{H}_{2} \mathrm{O}_{2}$. Besides participating in the typical enamine catalytic cycle, the chiral primary amine catalyst was found to work cooperatively with a ketone catalyst to activate $\mathrm{H}_{2} \mathrm{O}_{2}$ via an oxaziridine intermediate derived from in-situ generated ketimine intermediate. The resulted enamine-oxaziridine coupling then facilitated highly-controlled hydroxylation of $\beta$-ketocarbonyls that are not possible with other catalytic methods. The dual catalytic approach allows for highly enantioselective $\alpha$-hydroxylation of a broad range of $\beta$-ketocarbonyls. Particularly, late-stage hydroxylation for peptidyl amide or chiral esters can also
\end{abstract}


be achieved with high stereoselectivity. With its operational simplicity and mild conditions, this cooperative amine/ketone catalysis provides a new strategy in catalytic activation of $\mathrm{H}_{2} \mathrm{O}_{2}$ and expands the domain of typical amine and carbonyl catalysis to include those challenging transformations.

\section{Introduction}

Aminocatalysis is a fundamental activation mode in the transformations of carbonyl compounds. The catalysis has become a prevalent and enabling strategy for $\alpha$ - or $\beta$-functionalizations of carbonyls via enamine or iminium ion activation (Fig. 1a) ${ }^{1-2}$. On the other hand, carbonyl catalysis has recently appeared as a viable approach for the $\alpha$-functionalization of glycine-type amines ${ }^{3}$. Like its amine counterpart, carbonyl catalysis also bears a biological origin from Nature's enzyme, the pyridoxal-dependent aldolases ${ }^{4}$, and its prominent early successes can be traced back even before the renaissance of organocatalysis when chiral ketones or ketimines catalysts were extensively explored as metal-free oxidation catalysts ${ }^{5-7}$. In these cases, the carbonyl catalysts promoted concerted oxygen atom-transfer with olefins via dioxarine or oxaziridine intermediates and similar catalysis has been advanced to $\mathrm{C}-\mathrm{H}$ insertion reactions of alkanes lately ${ }^{8-9}$. Most of such ketone/aldehyde catalysts bear reactive carbonyls with electron-withdrawing substituents, and would readily couple with nucleophilic amine. Hence, as inherent reacting partners, amine and ketone/aldehyde carbonyl are mutually-exclusive for targeted catalytic transformations from a mechanistic point of view, like a yinyang interplay. A cooperative amine and carbonyl dual catalysis remains virtually unknown.

Widely applied in industry and environmental protection as a green terminal oxidant, hydrogen peroxide is considered as ideal oxygen source for chemical synthesis as it has the most active-oxygen content and is relatively safe and easy-to-handle with water as the sole byproduct ${ }^{10-12}$. Enantioselective C-H hydroxylation with $\mathrm{H}_{2} \mathrm{O}_{2}$ is arguably one of the most straightforward and atom-economic oxidation strategies in accessing 
chiral alcohols. However, to achieve catalytic asymmetric hydroxylation with hydrogen peroxide remains a great challenge and successful examples along this line are extremely scarce ${ }^{13-14}$. In order to modulate the activity and stereoselectivity under mild conditions, catalytic activations of $\mathrm{H}_{2} \mathrm{O}_{2}$ into more electrophilic oxygen species are required as $\mathrm{H}_{2} \mathrm{O}_{2}$ itself is less electrophilic and even serves as a good nucleophile under neutral and basic conditions ${ }^{15}$. Besides the established metal-mediated activation strategy ${ }^{12,14,16-17}$, there recently appeared organocatalytic strategy via dioxarine, oxaziridine or perhydrate intermediates with ketone

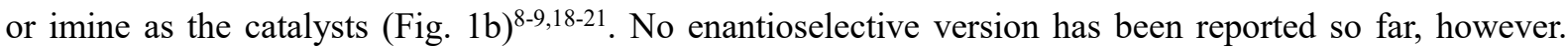
Herein, we reported the application of ketone catalysis in the asymmetric enamine-based $\alpha$-hydroxylation with hydrogen peroxide. Though enamine-based $\alpha$-oxygenation has been extensively explored, the successful examples relied on preactivated reagents such as oxaziridines, nitrosobenzenes, benzoyl peroxide and singlet oxygen $^{22-25}$. Catalytic direct enamine hydroxylation with hydrogen peroxide has not been achieved ${ }^{26}$. Ooi reported an enolate-based asymmetric $\alpha$-hydroxylation with hydrogen peroxide, unfortunately the reaction required a stoichiometric trichloroacetonitrile as an activating reagent ${ }^{27}$. Similar reactions have also been examined with chiral Lewis base catalysis but with moderate enantioselectivity and limited scopes ${ }^{28}$.

In our strategy (Fig. 1c), chiral primary amine catalyst worked in concert with a ketone catalyst to promote effectively $\alpha$-hydroxylation of $\beta$-ketocarbonyls with excellent stereocontrol beyond reach otherwise. The joint amine/ketone catalytic protocol could be applied in the late-stage hydroxylation reaction of complicated molecules. Mechanism studies revealed that the reaction proceeded via an enamine-oxaziridine coupling derived from the two working catalysts and both amine and ketone catalysts participated in activation of hydrogen peroxide via an iminium ion intermediate (Fig. 1c). 


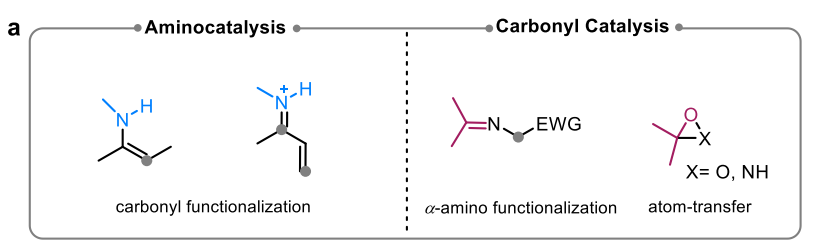

b
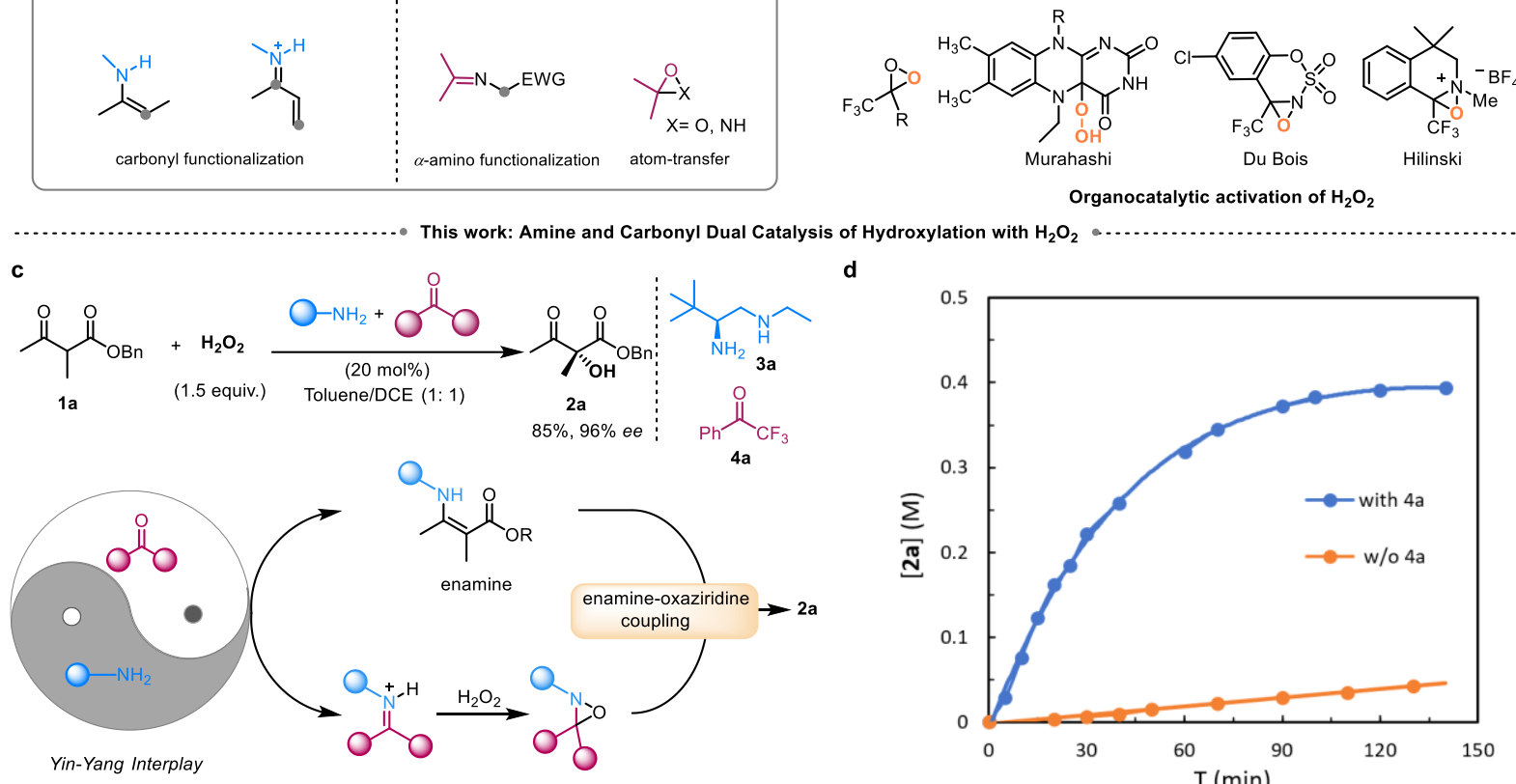

d

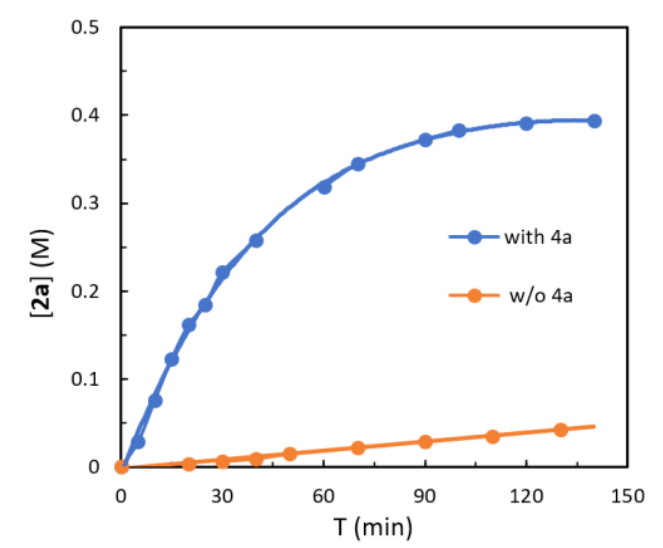

Figure 1. Organocatalytic activation of hydrogen peroxide. a, Typical activation modes in aminocatalysis and carbonyl catalysis. b, Active intermediates in organocatalytic activation of $\mathrm{H}_{2} \mathrm{O}_{2}$. c, Our concepts on amine and carbonyl dual catalysis: Chiral iminium ion in-situ generated by primary amine and ketone catalysts activates $\mathrm{H}_{2} \mathrm{O}_{2}$ in the form of chiral oxaziridine, which couples with enamine, also derived from amine catalysts to afford $\alpha$-hydroxylation product $\mathbf{2 a}$. $\mathbf{d}$, Kinetic profiles of ketone catalysis in the reaction of $1 \mathbf{a}$.

\section{Results}

Catalyst screening and reaction development. In our initial studies, we investigated the enantioselective hydroxylation of $\beta$-ketoester 1a with primary amine catalysts developed by our group. Among different primary amine catalysts screened, primary-secondary diamines such as $\mathbf{3 a}$ were identified as the preferred amine catalyst. The reaction with only amine catalyst (e.g. $\left.3 \mathbf{a} / \mathrm{Tf}_{2} \mathrm{NH}\right)$ was sluggish requiring at least 48 hours for complete conversion (Table 1, entry 3). At this point, the addition of a ketone catalyst was found to significantly enhance the reaction rate, and the optimal $\mathbf{3 a} / \mathbf{4 a}$ combination led to nearly 10 -times faster reaction (Fig. 1d). The reaction now gave $85 \%$ yield and 96\% ee in 3 hours (Table 1, entry 1). Aldehydes 
such as benzaldehyde was found to totally inhibit the reaction (Table 1, entry 9) and the smallest ketone, acetone showed diminished activity with maintaining enantioselectivity (Table 1, entry 10). Electronwithdrawing substitutions on the ketone carbonyl are critical to activity (Table 1, entries 11-13) and both $\mathrm{CF}_{3}$ (4a or trifluoroacetone) and carboxylate (4c) substituted ketones showed good activity with similar enantioselectivity. The primary-secondary diamine motif (e.g. 3a-3c) was critical to the joint catalysis as the reaction with tertiary amine (3d) showed rather low activity and enantioselectivity (Table 1, entries 14-16). In a control experiment, no reaction was observed in the absence of amine catalyst (Table 1, entry 2), pinpointing the decisive roles of aminocatalysis for the reaction. In consistent with well-known role of Br申nsted acid in facilitating aminocatalysis, the use of strong acid additive such as $\mathrm{Tf}_{2} \mathrm{NH}$ is essential for both the activity and enantioselectivity and low yield and enantioselectivity was obtained in its absence (Table 1, entry 4).

Other oxidants have also been examined in the dual catalytic system (Table 1, entries 5-8). The commonly employed oxidants such as $t$-butyl peroxide, oxone and air (or pure molecular oxygen) were virtually inactive and $m$-CPBA showed some activity but without any selectivity (Table 1, entry 6 ). These results indicated the current amine/ketone dual catalysis would preferentially activate $\mathrm{H}_{2} \mathrm{O}_{2}$ and facilitate the subsequent hydroxylation.

Table 1. Identifications of the amine/ketone dual catalysis and optimizations. 


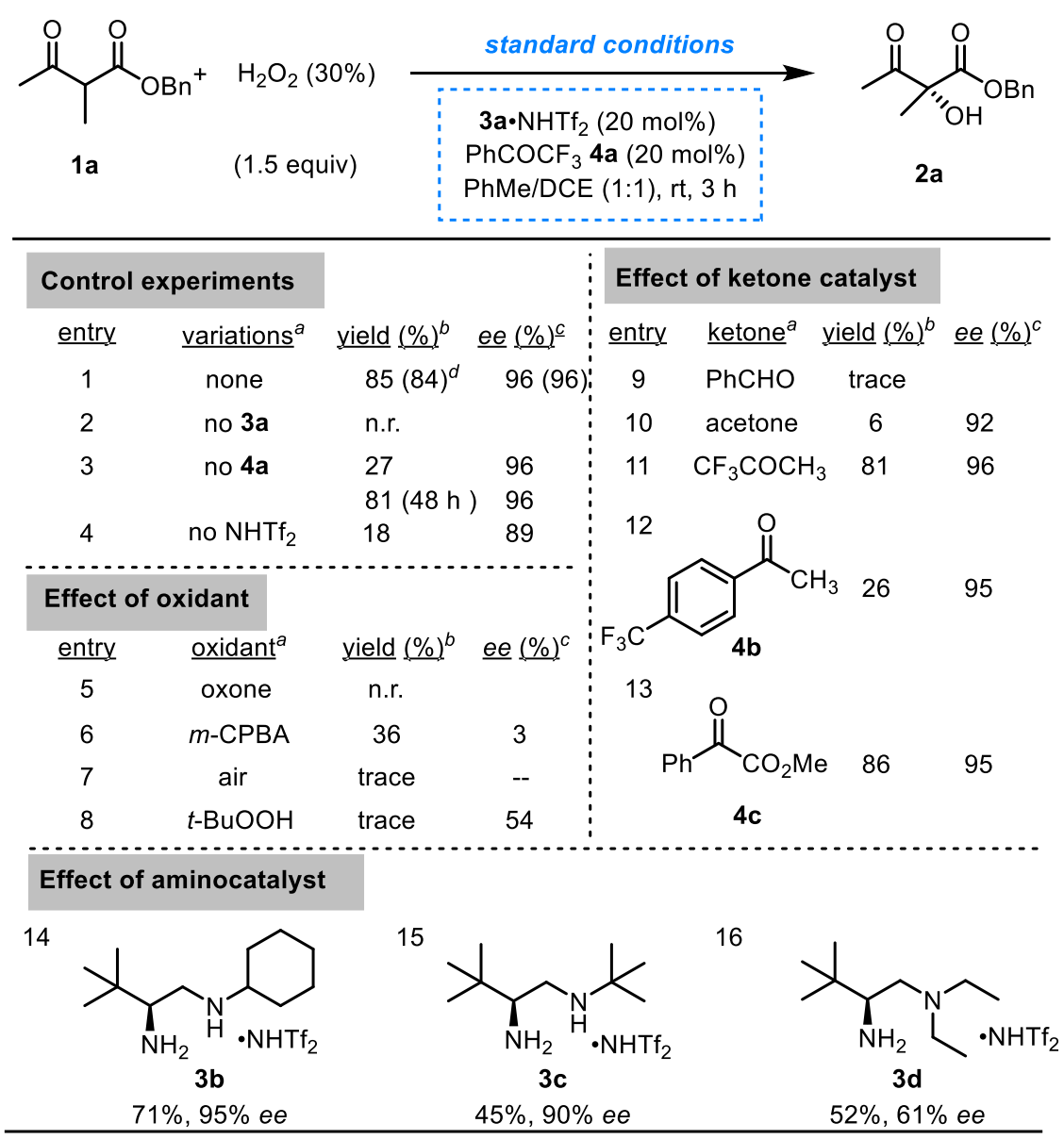

${ }^{a}$ Standard reaction condition: $\mathbf{1 a}(0.2 \mathrm{mmol})$, chiral amine $\mathbf{3 a} / \mathrm{NHTf}_{2}(20 \mathrm{~mol} \%), \mathrm{PhCOCF}_{3}(20 \mathrm{~mol} \%)$, and $\mathrm{H}_{2} \mathrm{O}_{2}$ (30 wt.\% in water, $0.3 \mathrm{mmol}$ ) in $0.5 \mathrm{~mL} \mathrm{PhMe} / \mathrm{DCE}(1 / 1)$ at room temperature in air for $3 \mathrm{~h} .{ }^{b}$ The yield was determined by GC analysis using 1, 3, 5-trimethoxybenzene as an internal standard. ${ }^{c}$ The $e e$ value was determined by HPLC analysis. ${ }^{d}$ Yield of isolated product for 4 hours.

Substrate scopes. As shown in Table 2, different ester groups of acetoacetates were well tolerated (Table 2, entries 1-8). A variety of $\alpha$-alkyl substituents ranging from methyl, ethyl to decanyl as well as benzyl all worked well to give the expected hydroxylation products with 70-94\% yields and 96-98\% ee (entries 9-13). Functional groups such as cyano (2n), ester (2o, 2p), acetal (2q), alkenyl (2r) and alkynyl (2s) at $\alpha$-position were equally applicable (entries 14-19). An ethyl ketone (2t) is also workable with 59\% yield and 96\% ee (entry 20). Cyclic ketoesters showed divergent behavior, while smaller rings $(\mathrm{n}=4,5,6)$ such as 
cyclopentanone or cyclohexanone did not show the expected reactivity, the larger cyclic ketoesters $(\mathrm{n}=7$, 12) reacted smoothly to give the desired products in high enantioselectivity with slightly diminished reactivity (entries 21-24). A gram-scale hydroxylation reaction of $\beta$-ketoester 1a was performed to probe the practicability with $10 \mathrm{~mol} \%$ catalyst loading and comparable isolated yield and enantioselectivity were obtained in $8 \mathrm{~h}$ (Table 2 , entry 1$)$.

$\beta$-Ketoamides are versatile structural motifs in biologically active compounds and their direct oxidative transformations are challenging because of their oxidative compatibility. It was found $\beta$-ketoamides worked extremely well under our dual catalytic conditions. Both aryl and alkyl amides could be incorporated to give the expected hydroxylation adducts with 93-99\% ee (entries 25-32). Aryl amides bearing either electrondonating group (6b), electron-withdrawing groups (6c-e) or streric ortho-substituent $(\mathbf{6 g})$ were equally applicable. Alkyl substituents at either $\alpha$ - or $\alpha$ '- position of ketoamides were well tolerated (entries 33-35). Cyclic ketoamides also worked well in the reactions to give the desired products in 55-87\% yields and 90 98\% ee (entries 36-38).

Table 2. Substrate scopes of amine/ketone dual catalysis ${ }^{a}$ 

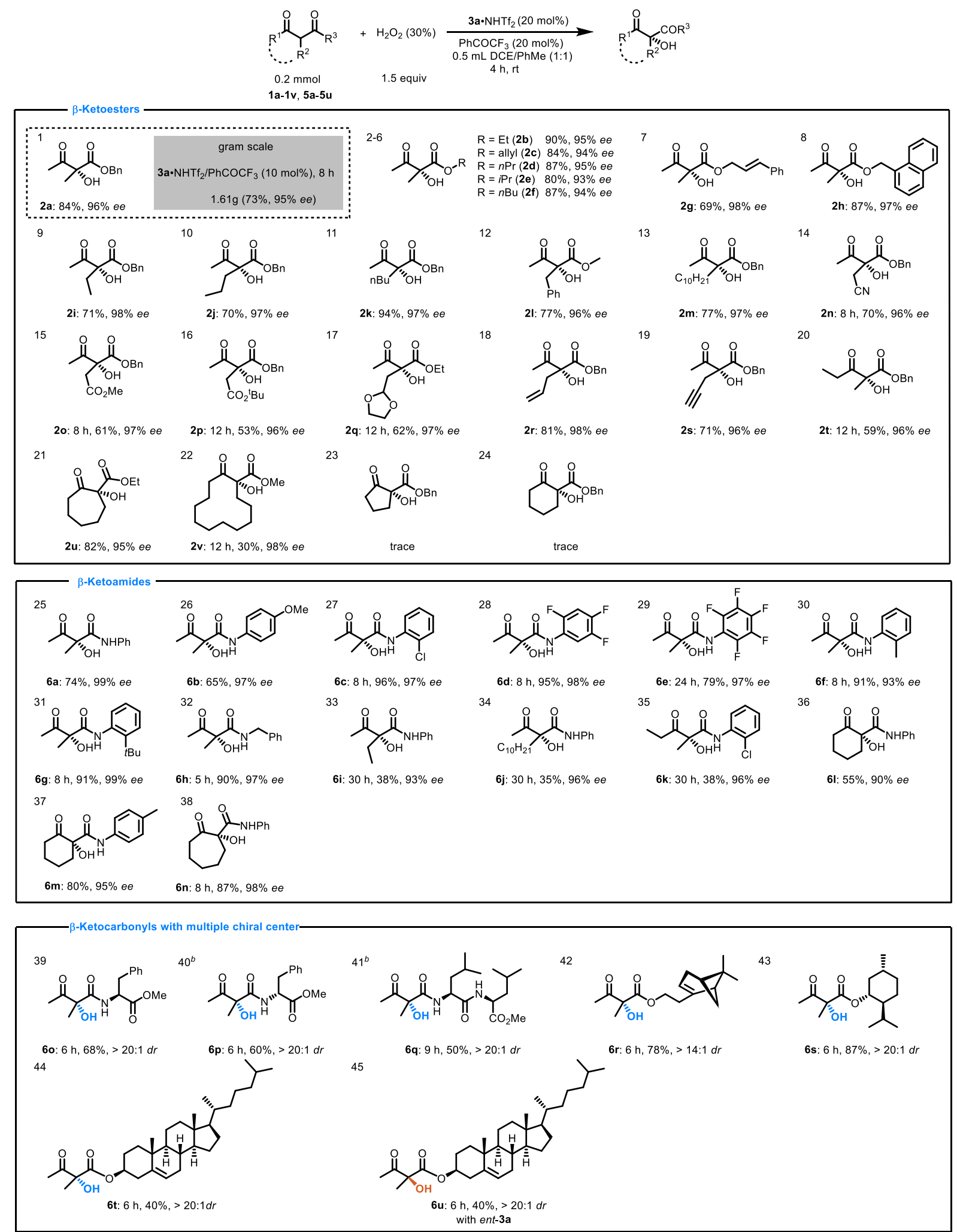

${ }^{a}$ Reactions were performed with $1(0.2 \mathrm{mmol}), 3 \mathrm{a} / \mathrm{Tf} \mathrm{NH}_{2} \mathrm{NH}(20 \mathrm{mo}), \mathrm{PhCOCF}_{3}\left(20 \mathrm{~mol}^{2}\right)$, and $\mathrm{H}_{2} \mathrm{O}_{2}(30 \mathrm{wt} \%$ in water, $0.3 \mathrm{mmol})$ in $0.5 \mathrm{~mL} \mathrm{PhMe} / \mathrm{DCE}(1 / 1)$ at room temperature in air for $4 \mathrm{~h}$. Yield of isolated product. Determined by HPLC analysis. ${ }^{b}$ The reaction was performed on scale of $0.1 \mathrm{mmol}$.

We also challenged the current protocol in the late-stage hydroxylation of structurally complexed substrates bearing existing chiral centers. In this regard, it was shown that the hydroxylation was solely catalyst-controlled to give the expected diastereoisomers with high diastereoselectivity (entry 39 vs entry 40 , 
entry $44 v s$ entry 45). Peptidyl amide (6q) or nopyl (6r) and menthyl esters (6s) worked smoothly. The catalysis also worked with a cholesteryl ester $(\mathbf{6 t}$ and $\mathbf{6 u})$ in reasonably good activity and high diastereoselectivity (entries 44 and 45).

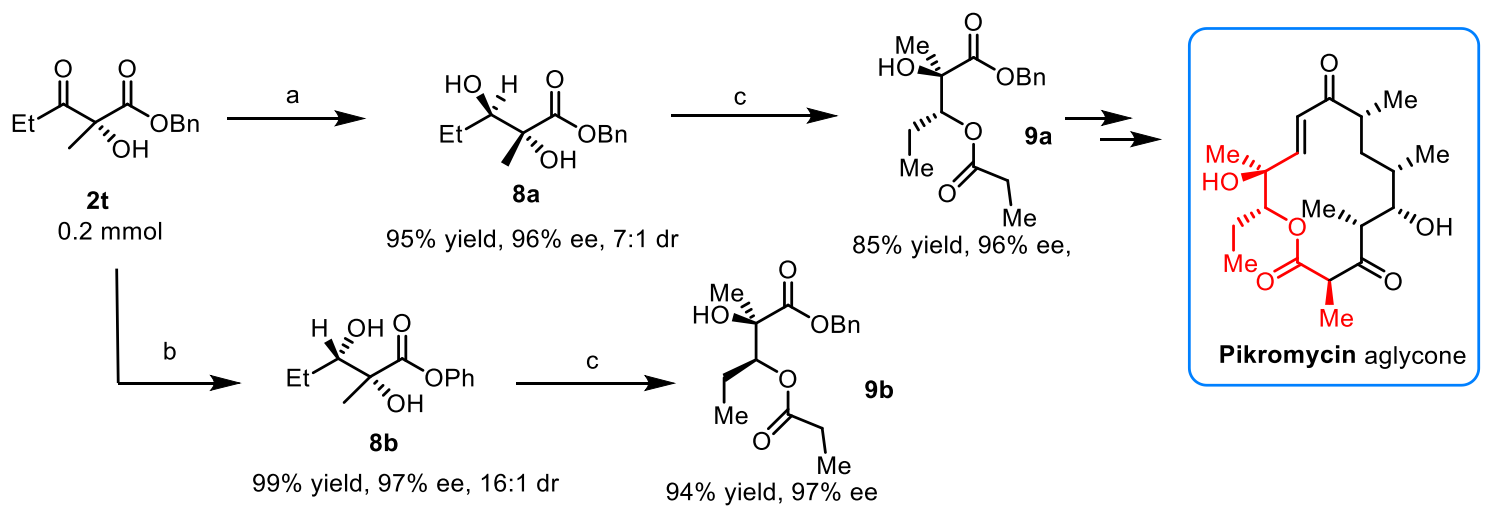

Figure 2. Synthetic application. Conditions: a) $\mathrm{NaBH}(\mathrm{OAc})_{3}$ (1 equiv), $\mathrm{HOAc}(5 \mathrm{~mol} \%), \mathrm{DCM}, 0{ }^{\circ} \mathrm{C}, 30$ min. b) $\mathrm{ZnCl}_{2}$ (1 equiv), $\mathrm{NaBH}_{4}$ (1 equiv), THF, $-40{ }^{\circ} \mathrm{C}, 30$ min. c) Acetic anhydride, $\mathrm{DMAP}, \mathrm{Et}_{3} \mathrm{~N}$.

Macrolide antibiotics are significantly important natural product that are wildly prescribed to treat bacterial infections. The $\alpha$-hydroxy-carbonyl moiety in this family of antibiotics, e.g. Pikromycin, inspired us to pursue a concise synthetic path ${ }^{29}$. The key intermediate $\mathbf{2 t}$ could by (be) synthesized by the developed catalytic asymmetric hydroxylation, and reduction of the keto moiety of $\mathbf{2 t}$ followed by chemoselective acylation afforded the desired 1,2-anti-diol fragment 9a. Changing the reducing condition led to a reversed configuration of 1,2-diol, producing 1,2-syn-diol 9b (Fig. 2), which is also a versatile synthon in natural product synthesis ${ }^{30}$.

Mechanism studies. To account for the dramatic promoting effect of $\mathbf{4 a}$, a $\mathrm{H}_{2} \mathrm{O}_{2}$-activation mechanism was invoked. As known, the organocatalytic activation of $\mathrm{H}_{2} \mathrm{O}_{2}$ may proceed via ketone mode with a dioxirane intermediate (II, derived from I, Fig. 3a) or ketimine mode with an oxaziridine intermediate IV (derived from III, Fig. 3a). ${ }^{18} \mathrm{O}$-Labelling studies with $\mathrm{H}_{2}{ }^{18} \mathrm{O}-\mathrm{UHP}$ was conducted. Under the bifunctional

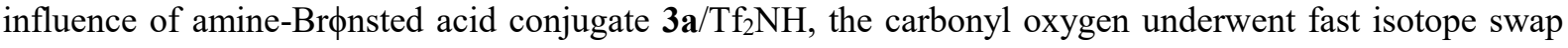


with $\mathrm{H}_{2}{ }^{18} \mathrm{O}$ and double ${ }^{18} \mathrm{O}$-labelling product on both the carbonyl-O and $\alpha$-hydroxyl-O would be formed if the reaction followed the dioxirane-II pathway (Fig. 3b). However, such a doubly-labelled product was not detected by in-situ HRMS analysis. In addition, control experiment indicated that preformed imine such as 4d could effectively promote the reaction with $47 \%$ yield and $95 \%$ ee (Fig. 3c). The reaction with a prepared oxaziridine $4 \mathbf{e}$ worked well to give the expected adduct with comparable enantioselectivity (Fig. 3d). Taken together, these results suggested that the reaction would preferentially proceed via the ketimine-pathway with III or IV, the ketone pathway via I or II, even if not entirely excluded, should be neglectable, to note that dioxirane formation between ketone and $\mathrm{H}_{2} \mathrm{O}_{2}$ normally required either strong acid or strong basic conditions $^{12}$ and amine-ketone coupling would be favored over perhydroxylation of ketone under the present acid-base bifunctional conditions. Nano-ESI-MS analysis of the reaction mixture led to the identification of the expected iminium ion intermediate $\mathbf{9 b}$ and the oxaziridine intermediate IV' (Fig. 4a) and the structure of IV' was further established by collision-induced dissociation analysis (Fig. 4b), adding direct evidence to a ketimine mechanism. 


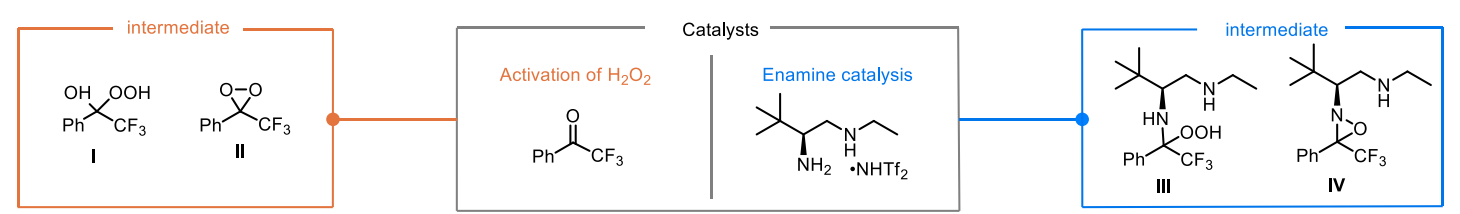

b
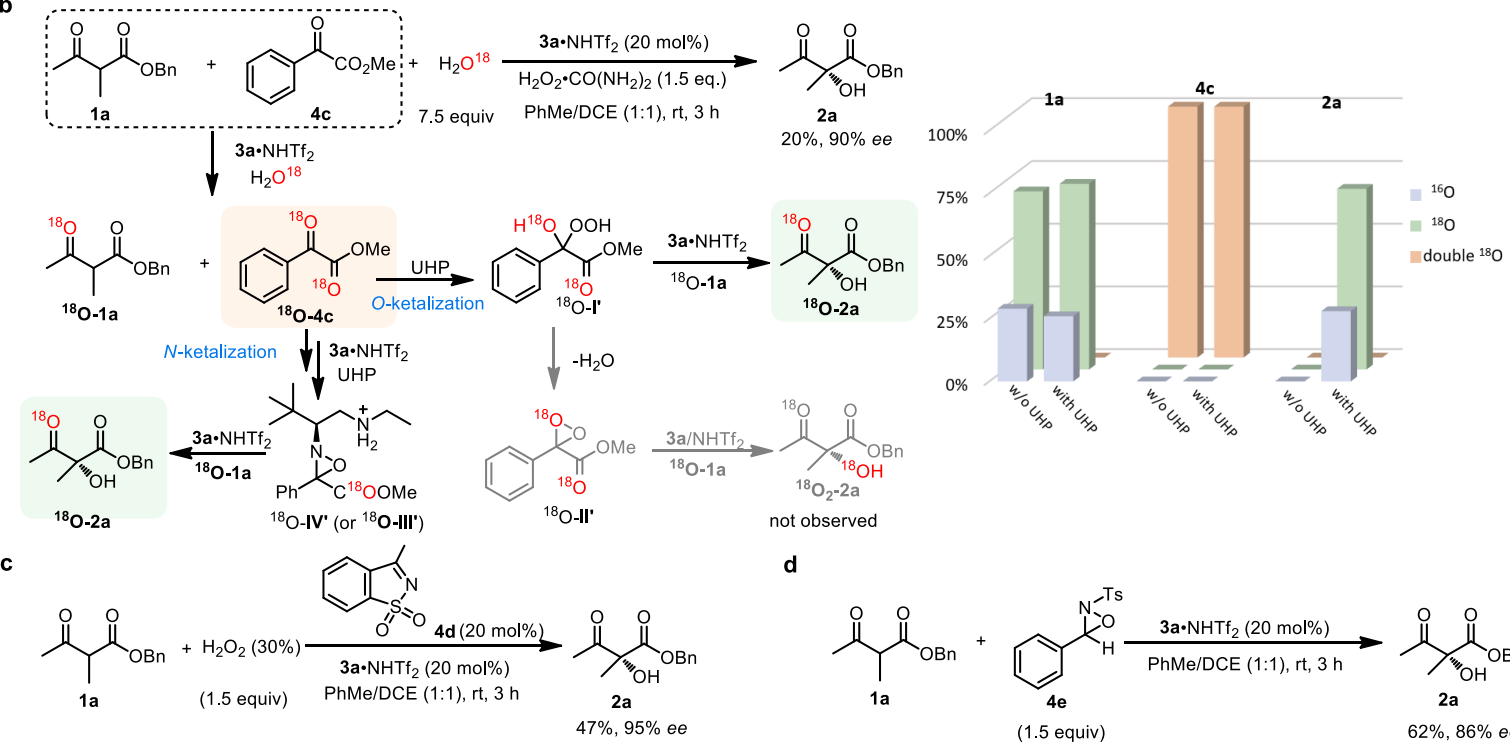

d
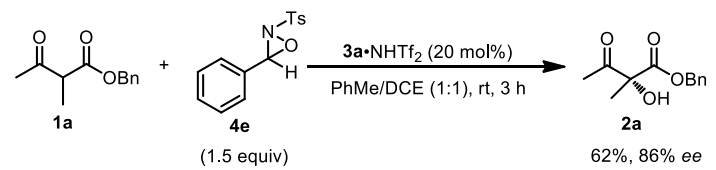

Figure 3. a, The possible oxidative intermediate for the ketone pathway (left) and ketimine pathway (right).

b, ${ }^{18} \mathrm{O}$ Labeling experiments by $\mathrm{H}_{2}{ }^{18} \mathrm{O}$. The possible labelling pathways were listed on the left with experimental observations showing on the right. The only pathway leading to doubly labelled product ${ }^{18} \mathrm{O}_{2^{-}}$ 2 was through a dioxirane intermediate ${ }^{18} \mathrm{O}-\mathrm{II}$ ', and the absence of ${ }^{18} \mathrm{O}_{2}-\mathbf{2}$ suggested this was unlikely. c, Control experiment with $\mathbf{4 a}$ as a catalyst, conditions: $\mathbf{1 a}(0.2 \mathrm{mmol}), \mathbf{3 a} / \mathrm{Tf}_{2} \mathrm{NH}(20 \mathrm{~mol} \%), \mathbf{4 d}(20 \mathrm{~mol} \%)$, and $\mathrm{H}_{2} \mathrm{O}_{2}$ (30 wt.\% in water, $\left.0.3 \mathrm{mmol}\right)$ in $0.5 \mathrm{~mL} \mathrm{PhMe} / \mathrm{DCE}(1 / 1)$ at room temperature in air for $3 \mathrm{~h}$. d, Control experiment with preformed oxaziridine as the oxidant, conditions: $\mathbf{1 a}(0.2 \mathrm{mmol}), \mathbf{3 a} / \mathrm{Tf}_{2} \mathrm{NH}(20$ mol\%) and oxaziridine $4 \mathrm{e}(0.30 \mathrm{mmol})$ in $0.5 \mathrm{~mL} \mathrm{PhMe} / \mathrm{DCE}(1 / 1)$ at room temperature in air for $3 \mathrm{~h}$. 


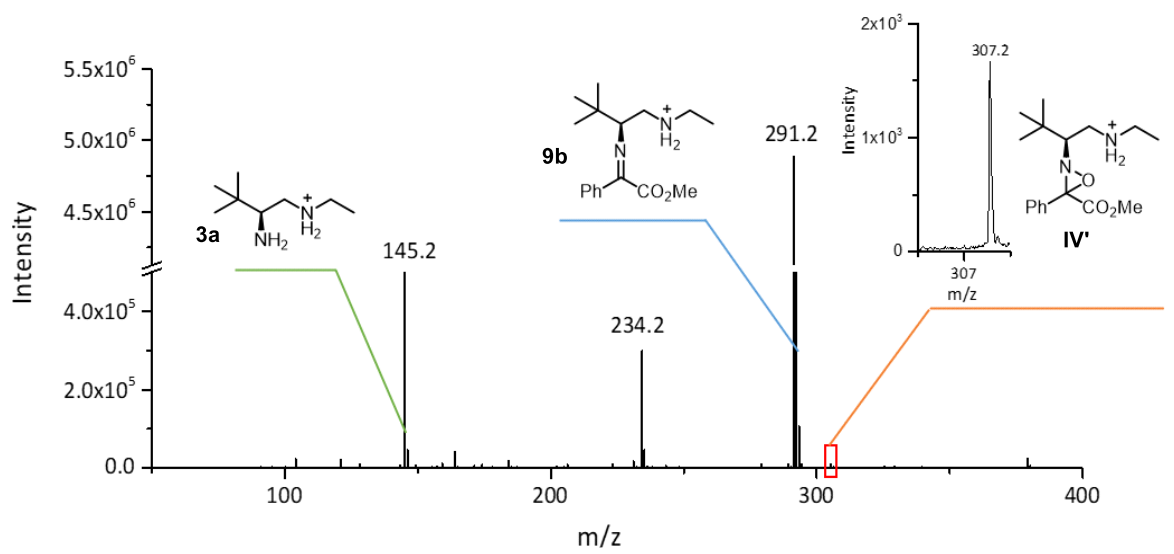

b

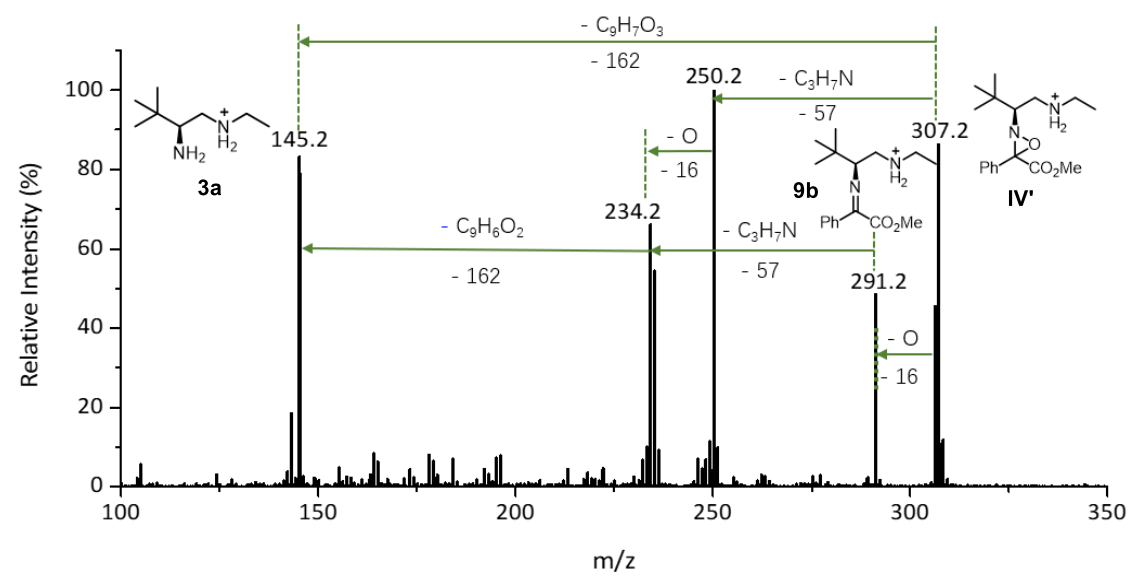

Figure 4. a, nESI(+)-MS spectrum of reaction of $\mathbf{3 a} / \mathrm{NHTf}_{2}, \mathbf{4} \mathbf{c}$ and $\mathrm{H}_{2} \mathrm{O}_{2}$ (30 wt.\% in water) in DCE/PhMe in situ. b, CID analysis of oxaziridine intermediate IV'.

The kinetics was determined by measuring the initial rates with varying concentration of $\mathrm{H}_{2} \mathrm{O}_{2}$, substrate or catalysts (Supplementary Section 8). The reaction was found to be zero-order on either substrate 1a or $\mathrm{H}_{2} \mathrm{O}_{2}$ (Fig. 5, a-b) and first-order on both amine and ketone catalysts (Fig. 5, c-d). A rate-limiting state preceding the $\mathrm{C}-\mathrm{O}$ bond formation, is in line with this kinetic scenario. The non-linear effect (NLE) of the dual catalytic system was also determined and a minor negative NLE was clearly noted (Fig. 5e). Previously, negative NLE was reported in proline-catalyzed Robinson annulation by Agami ${ }^{31-32}$, however, the existence of NLE in this reaction was later disapproved by List and Houk ${ }^{33}$. The observation of (-)-NLE in our reaction suggests the stereo-determining step is not a one-catalyst system. An enamine-oxaziridine coupling involving 
two molecules of chiral aminocatalysts can be proposed to account for the NLE (Fig. 5f). Similar two-catalyst mode has been proposed by Kagan and $\mathrm{Agami}^{31}$, and in this mode the reaction with the hetero- $R / S$ and $S / R$ combination is favored over that with homo- $R / R$ or $S / S$ combination. On these bases, a dual catalytic pathway involving an enamine-cycle and a ketimine cycle was proposed as shown in Fig. 5g. In this coupled cycle, ketimine formation from 3a and $\mathbf{4 a}$ is rate-limiting step, and the effective coupling between enamine $\mathbf{1 0}$ and intermediate III or IV leads to the hydroxylated adduct in high stereoselectivity.

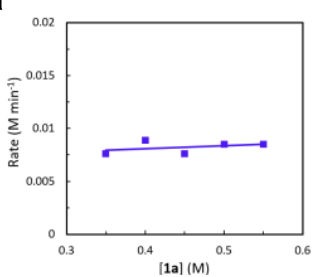

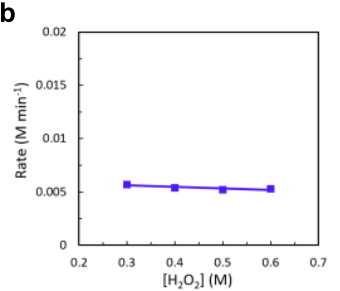

c

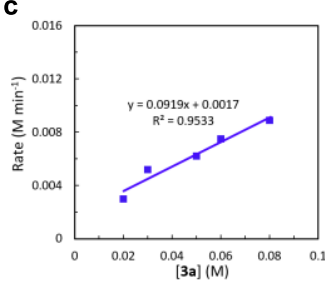

d

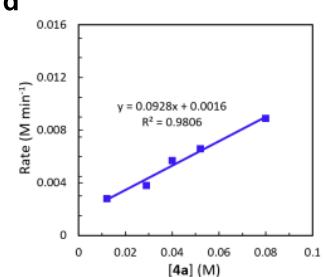

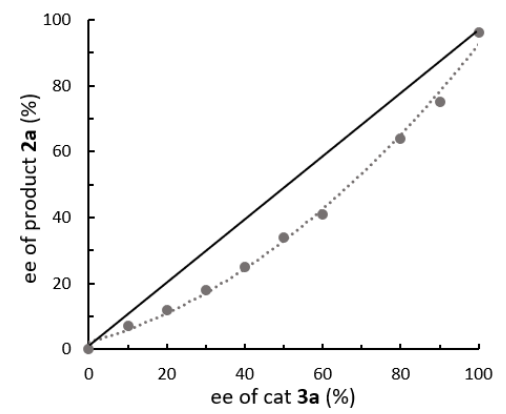

$\mathbf{f}$

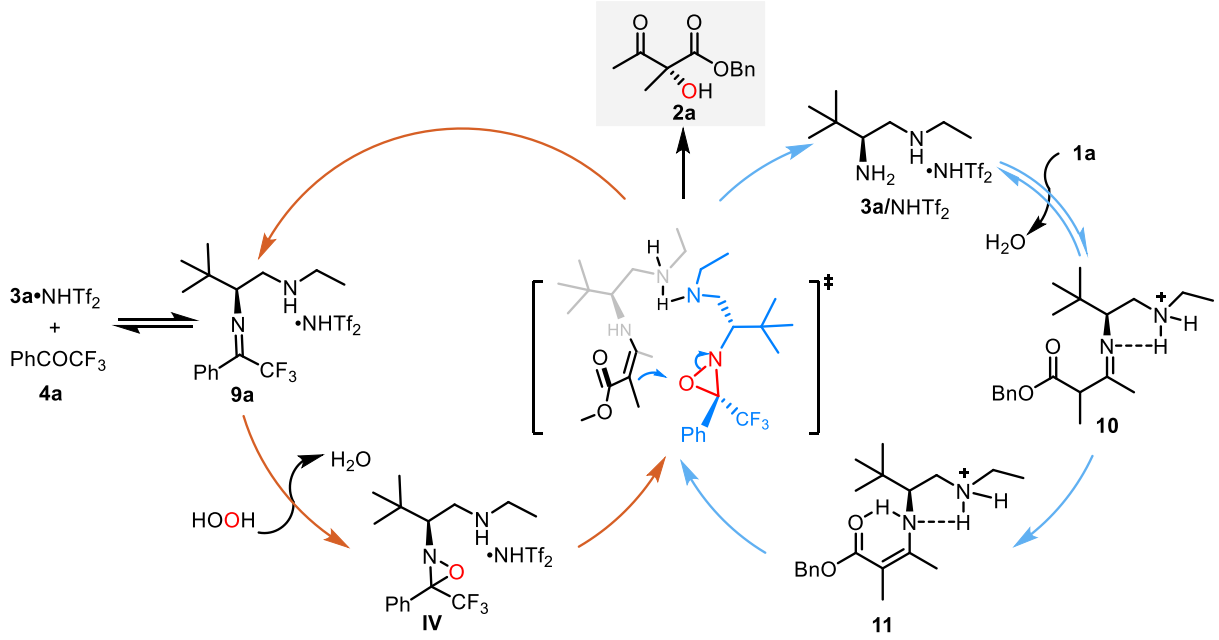

Figure 5. a-d, Kinetic order plots for $\mathbf{1 a}, \mathrm{H}_{2} \mathrm{O}_{2}, \mathbf{3 a}$ and $\mathbf{4 a}$, respectively. Plot of initial rate against [1a] (from 
0.35 to $0.55 \mathrm{M}$ in DCE/PhMe) (a), $\left[\mathrm{H}_{2} \mathrm{O}_{2}\right]$ (from 0.3 to $0.6 \mathrm{M}$ in DCE/PhMe) (b), [3a] (from 0.02 to $0.08 \mathrm{M}$ in DCE/PhMe) (c) and [4a] (from 0.01 to $0.08 \mathrm{M}$ in DCE/PhMe) (d). e, Plot of enantiomeric excess of $\mathbf{2 a}$ against the enantiomeric excess of 3a, showing the existence of negative nonlinear effect (NLE). $\mathbf{f}$, Twocatalyst model for the negative NLE with the hetero-combinations R/S and S/R reacting faster than homocombination R/R and S/S, attenuating the enantioselectivity. g, Proposed catalytic cycle of synergetic catalysis of primary amine $\mathbf{3 a}$ and $\mathrm{PhCOCF}_{3} \mathbf{4 a}$.

We further verified the reaction profile by DFT calculation (Fig. 6, see Supplementary Section 9 for details). Both the enamine (Int3) and ketimine (Int5) formation followed a bifunctional mode with the protonated secondary amine serving as the acid catalytic moiety, characteristic of the diamine-Bronsted acid catalysis as known ${ }^{34}$. In this coupled cycles, aminocatalyst $\mathbf{3 a} / \mathrm{NHTf}_{2}$ played a dual role in reacting with either ketoester 1a or ketone catalyst 4a to form the key enamine (Int3) and oxaziridine (IV) intermediates, respectively. The ketalization with 4a or 1a are quickly equilibrated processes, the subsequent dehydration of ketal Int4 is much more endergonic than Int1, making the former an overall rate-limiting step with an energy barrier of $20.9 \mathrm{kcal} / \mathrm{mol}$. The down-hill re-ketalization of Int5 with hydrogen peroxide is quite facile to give the expected perhydroxyl acetal III and oxaziridine IV. The conversion of III to IV occurred spontaneously with no obvious barrier. Both III and IV could effectively couple with the enamine intermediate Int3 to give $R$ selective product via TS9 (Supplementary Fig. 5) and TS7, respectively. The minor S-product was formed by the $E$-enamine addition to $\mathbf{I V}$, with a calculated $99 \%$ ee value, which is in accordance with the experimental result. In TS7, an intermolecular N-H-N hydrogen bonding between two secondary amines side chains was noted, facilitating the alignment of the two reactive intermediates. Depending on the ionic status of the two intermediates, anion mediated H-bonding may also contribute and such a ternary TS8 could also be located, showing a slightly favored energy barrier of $18.4 \mathrm{kcal} / \mathrm{mol}$ (Fig. 6) ${ }^{35}$. 


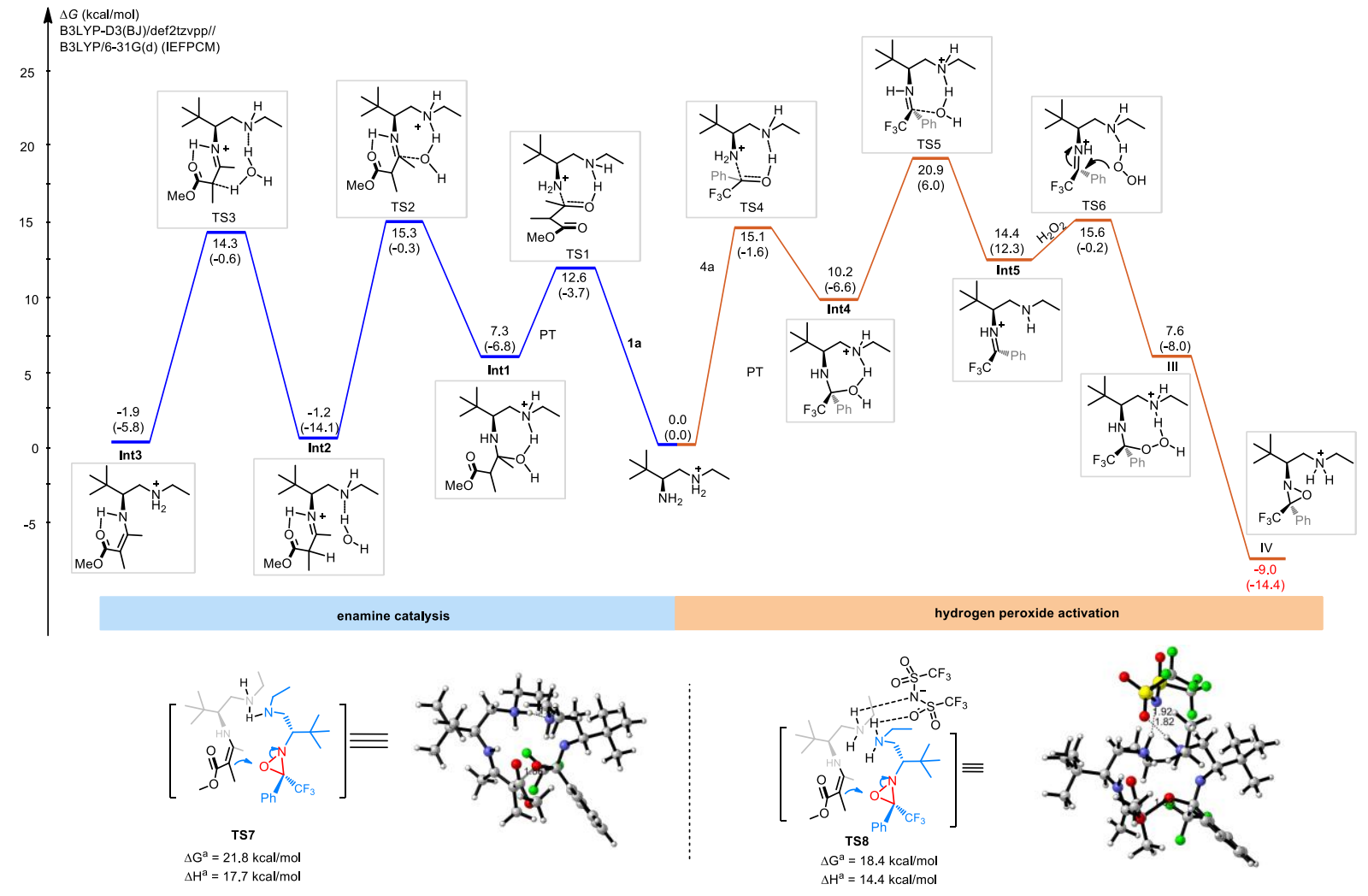

Figure 6. DFT-Calculated free-energy profiles on the activation of hydrogen peroxide and formation of enamine and the key TSs for the hydroxylation step.

Conclusion. In summary, we have shown that amine and ketone can work in concert to promote effectively enantioselective transformation of carbonyls. The dual amine and ketone catalysis enable an electrophilic activation of $\mathrm{H}_{2} \mathrm{O}_{2}$ via in-situ generated ketimine in the form of oxaziridine and allows for its effective coupling with a nucleophilic enamine intermediate. The developed dual organocatalytic protocol demonstrated high activity and enantioselectivity for a broad range of $\beta$-ketoeasters and $\beta$-ketoamides that are not possible with other catalytic approaches. The current approach represents a new organocatalytic strategy in activating hydrogen peroxide. Given its versatility and operational simplicity, further advances along this line are anticipated.

\section{Methods}

Here we describe the general procedures for the $\alpha$-hydroxylation of carbonyl compounds through dual amine 
and ketone catalysis.

General procedure for conditions: An oven-dried tube equipped with stir bar was charged with corresponding $\beta$-ketocarbonyls (1, $0.2 \mathrm{mmol})$ and aminocatalyst 3a/ $\mathrm{NHTf}_{2}(20 \mathrm{~mol} \%)$. After dissolution in a mixed solvent of toluene and 1,2-dichloroethane $(0.5 \mathrm{~mL}, 1: 1, \mathrm{v} / \mathrm{v})$, trifluoroacetophenone $4 \mathrm{a}(20 \mathrm{~mol} \%)$ was added to the vial. Then $\mathrm{H}_{2} \mathrm{O}_{2}$ ( 30 wt.\% in water, $0.3 \mathrm{mmol}$ ) was added via a syringe. Upon completion of the addition, the reaction was stirred at room temperature for at least 4 hours (TLC analysis). The solvent was removed under reduced pressure and the residue was purified by silica gel chromatography (petroleum ether/ethyl acetate $=$ 20:1-4:1) to afford the desired product $\mathbf{2}$ or $\mathbf{6}$. The enantiomeric excess was determined by HPLC.

\section{Acknowledgements}

We thank the Natural Science Foundation of China (21672217, 21861132003 and 22031006) and Tsinghua University Initiative Scientific Research Program for financial support. Tsinghua Xuetang Talents Program is thanked for computational support. S.L. is supported by the National Program of Top-notch Young Professionals. We thank Professor Xinhao Zhang and Heming Jiang from Peking University Shenzhen Graduate School for the assistance in ESI-MS studies.

\section{Author Information}

\section{Corresponding Authors}

Sanzhong Luo - Center of Basic Molecular Science, Department of Chemistry, Tsinghua University, Beijing 100084, China; Beijing National Laboratory for Molecular Sciences, Key Laboratory for Molecular Recognition and Function, Institute of Chemistry, Chinese Academy of Sciences, Beijing 100190, China Email:luosz@tsinghua.edu.cn

Long Zhang - Center of Basic Molecular Science, Department of Chemistry, Tsinghua University, Beijing 100084, China; Email: zhanglong@tsinghua.edu.cn 


\section{Authors}

Mao Cai - Beijing National Laboratory for Molecular Sciences, Key Laboratory for Molecular Recognition and Function, Institute of Chemistry, Chinese Academy of Sciences, Beijing 100190, China; School of Chemical Science, University of Chinese Academy of Sciences, Beijing 100049, China

Kaini Xu - Center of Basic Molecular Science, Department of Chemistry, Tsinghua University, Beijing 100084, China

Yuze Li - Beijing National Laboratory for Molecular Sciences, Key Laboratory for Analytical Chemistry for Living Biosystems, Institute of Chemistry, Chinese Academy of Sciences, Beijing 100190, China; School of Chemical Science, University of Chinese Academy of Sciences, Beijing 100049, China

Zongxiu Nie - Beijing National Laboratory for Molecular Sciences, Key Laboratory for Analytical Chemistry for Living Biosystems, Institute of Chemistry, Chinese Academy of Sciences, Beijing 100190, China; School of Chemical Science, University of Chinese Academy of Sciences, Beijing 100049, China

\section{Contributions}

S.L. designed and supervised the project. M.C. and K.X. performed the experimental studies; M.C. conducted the mechanism studies with the assistance of Z.N. and Y.L. providing ESI-MS analysis; L.Z. conducted DFT calculation. S.L., M.C. and L.Z. wrote the manuscript.

\section{Data availability}

All the data supporting the findings of this study are available within the paper and its Supplementary Information files. The datasets generated during and/or analysed during the current study are available from the corresponding author on reasonable request.

\section{Ethics declarations}

Competing interests 
The authors declare no competing interests.

\section{Additional information}

\section{References}

1. Mukherjee, S., Yang, J. W., Hoffmann, S. \& List, B. Asymmetric enamine catalysis. Chem. Rev. 107, $5471-5569$ (2007).

2. Matos Paz, B., Jiang, H. \& Jørgensen, K. A. Aminocatalysis: Beyond steric shielding and hydrogenbonding. Chem. - Eur. J. 21, 1846- 1853 (2015).

3. Chen, J. et al. Carbonyl catalysis enables a biomimetic asymmetric Mannich reaction. Science 360, 1438$1442(2018)$.

4. Dückers, N., Baer, K., Simon, S., Gröger, H. \& Hummel, W. Threonine aldolases-screening, properties and applications in the synthesis of non-proteinogenic $\beta$-hydroxy- $\alpha$-amino acids. Appl. Microbiol. Biotechnol. 88, 409-424 (2010).

5. Adam, W., Saha-Möller, C. R. \& Ganeshpure, P. A. Synthetic applications of nonmetal catalysts for homogeneous oxidations. Chem. Rev. 101, 3499-3548 (2001).

6. Shi, Y. Organocatalytic asymmetric epoxidation of olefins by chiral ketones. Acc. Chem. Res. 37, 488-496 (2004).

7. Wong, O. A. \& Shi, Y. Organocatalytic oxidation. Asymmetric epoxidation of olefins catalyzed by chiral ketones and iminium salts. Chem. Rev. 108, 3958-3987 (2008).

8. Brodsky, B. H. \& Du Bois, J. Oxaziridine-mediated catalytic hydroxylation of unactivated $3^{\circ} \mathrm{C}-\mathrm{H}$ bonds using hydrogen peroxide. J. Am. Chem. Soc. 127, 15391-15393 (2005).

9. Pierce, C. J. \& Hilinski, M. K. Chemoselective hydroxylation of aliphatic $s p^{3} \mathrm{C}-\mathrm{H}$ bonds using a ketone catalyst and aqueous $\mathrm{H}_{2} \mathrm{O}_{2}$. Org. Lett. 16, 6504-6507 (2014). 
10. Campos-Martin, J. M., Blanco-Brieva, G. \& Fierro, J. L. G. Hydrogen peroxide synthesis: An outlook beyond the anthraquinone process. Angew. Chem. Int. Ed. 45, $6962-6984$ (2006).

11. Russo, A., De Fusco, C. \& Lattanzi, A. Organocatalytic asymmetric oxidations with hydrogen peroxide and molecular oxygen. ChemCatChem 4, 901 - 916 (2012).

12. Bryliakov, K. P. Catalytic asymmetric oxygenations with the environmentally benign oxidants $\mathrm{H}_{2} \mathrm{O}_{2}$ and O . Chem. Rev. 117, 11406-11459 (2017).

13. Newhouse, T. \& Baran, P. S. If C-H bonds could talk: Selective C-H bond oxidation. Angew. Chem. Int. Ed. 50, $3362-3374$ (2011).

14. Milan, M., Bietti, M. \& Costas, M. Enantioselective aliphatic $\mathrm{C}-\mathrm{H}$ bond oxidation catalyzed by bioinspired complexes. Chem. Commun. 54, 9559-9570 (2018).

15. Mayer, R. J. et al. Solvation accounts for the counterintuitive nucleophilicity ordering of peroxide anions. Angew. Chem. Int. Ed. 56, $13279-13282$ (2017).

16. White, M. C. \& Zhao, J. Aliphatic C-H oxidations for late-stage functionalization. J. Am. Chem. Soc. 140, 13988-14009 (2018).

17. Cianfanelli, M. et al. Enantioselective $\mathrm{C}-\mathrm{H}$ lactonization of unactivated methylenes directed by carboxylic acids. J. Am. Chem. Soc. 142, 1584-1593 (2020).

18. Murahashi, S.-I., Oda, T. \& Masui, Y. Flavin-catalyzed oxidation of amine and sulfur compounds with hydrogen peroxide. J. Am. Chem. Soc. 111, 5002-5003 (1989).

19. Brodsky, B. H. \& Du Bois, J. Oxaziridine-mediated catalytic hydroxylation of unactivated $3^{\circ} \mathrm{C}-\mathrm{H}$ bonds using hydrogen peroxide. J. Am. Chem. Soc. 127, 15391-15393 (2005).

20. Wang, D., Shuler, W. G., Pierce, C. J. \& Hilinski, M. K. An iminium salt organocatalyst for selective aliphatic C-H hydroxylation. Org. Lett. 18, 3826-3829 (2016).

21. Peris, G., Jakobsche, C. E. \& Miller, S. J. Aspartate-catalyzed asymmetric epoxidation reactions. J. Am. 
Chem. Soc. 129, 8710-8711 (2007).

22. Witten, M. R. \& Jacobsen, E. N. A simple primary amine catalyst for enantioselective $\alpha$-hydroxylations and $\alpha$-fluorinations of branched aldehydes. Org. Lett. 17, 2772-2775 (2015).

23. Brown, S. P., Brochu, M. P., Sinz, C. J. \& MacMillan, D. W. C. The direct and enantioselective organocatalytic $\alpha$-oxidation of aldehydes. J. Am. Chem. Soc. 125, 10808-10809 (2003).

24. Wang, D., Xu, C., Zhang, L. \& Luo, S. Asymmetric $\alpha$-benzoyloxylation of $\beta$-ketocarbonyls by a chiral primary amine catalyst. Org. Lett. 17, 576-579 (2015).

25. Córdova, A., Sundén, H., Engqvist M., Ibrahem, I. \& Casas J. The direct amino acid-catalyzed asymmetric incorporation of molecular oxygen to organic compounds. J. Am. Chem. Soc. 126, 8914-8915 (2004).

26. Mairhofer, C., Novacek, J. \& Waser, M. Synergistic ammonium (hypo)iodite/imine catalysis for the asymmetric $\alpha$-hydroxylation of $\beta$-ketoesters. Org. Lett. 22, 6138-6142 (2020).

27. Ohmatsu, K., Ando, Y. \& Ooi, T. In situ electrophilic activation of hydrogen peroxide for catalytic asymmetric $\alpha$-hydroxylation of 3-substituted oxindoles. Synlett 28, 1291-1294 (2017).

28. Li, Z., Lian, M., Yang, F., Meng, Q. \& Gao, Z. Diterpenoid alkaloid lappaconine derivative catalyzed asymmetric $\alpha$-hydroxylation of $\beta$-dicarbonyl compounds with hydrogen peroxide. Eur. J. Org. Chem. 2014, 3491-3495 (2014).

29. Trost, B. M., Malhotra, S., Koschker, P. \& Ellerbrock, P. Development of the enantioselective addition of ethyl diazoacetate to aldehydes: Asymmetric synthesis of 1,2-diols. J. Am. Chem. Soc. 134, 2075-2084 (2012).

30. Truong, P. M., Zavalij, P. Y. \& Doyle, M. P. Highly enantioselective carbonyl-ene reactions of 2,3diketoesters: efficient and atom-economical process to functionalized chiral $\alpha$-hydroxy- $\beta$-ketoesters. Angew. Chem. Int. Ed. 53, 6468-6472 (2014). 
31. Puchot, C., Samuel, O., Duñach, E., Zhao, S., Agami, C. \& Kagan, B. Nonlinear effects in asymmetric synthesis. Examples in asymmetric oxidations and aldolization reactions. J. Am. Chem. Soc. 108, 2353-2357 (1986).

32. Puchot, C. \& Agami, C. Kinetic analysis of the dual catalysis by proline in the asymmetric intramolecular aldol reaction. J. Mol. Catal. 38, 341-343 (1986).

33. Hoang, L., Bahmanyar, S., Houk, K. N. \& List, B. Kinetic and stereochemical evidence for the involvement of only one proline molecule in the transition states of proline-catalyzed intra- and intermolecular aldol reactions. J. Am. Chem. Soc. 125, 16-17 (2003).

34. Zhang, L., Fu, N. \& Luo, S. Pushing the limits of aminocatalysis: Enantioselective transformations of $\alpha$-branched $\beta$-ketocarbonyls and vinyl ketones by chiral primary amines. Acc. Chem. Res. 48, 986-997 (2015).

35. Shokri, A., Ramezani, M., Fattahi, A. \& Kass, R. S. Electrostatically defying cation-cation clusters: Can likes attract in a low-polarity environment? J. Phys. Chem. A 117, 9252-9258 (2013).

\section{Graphic abstract}

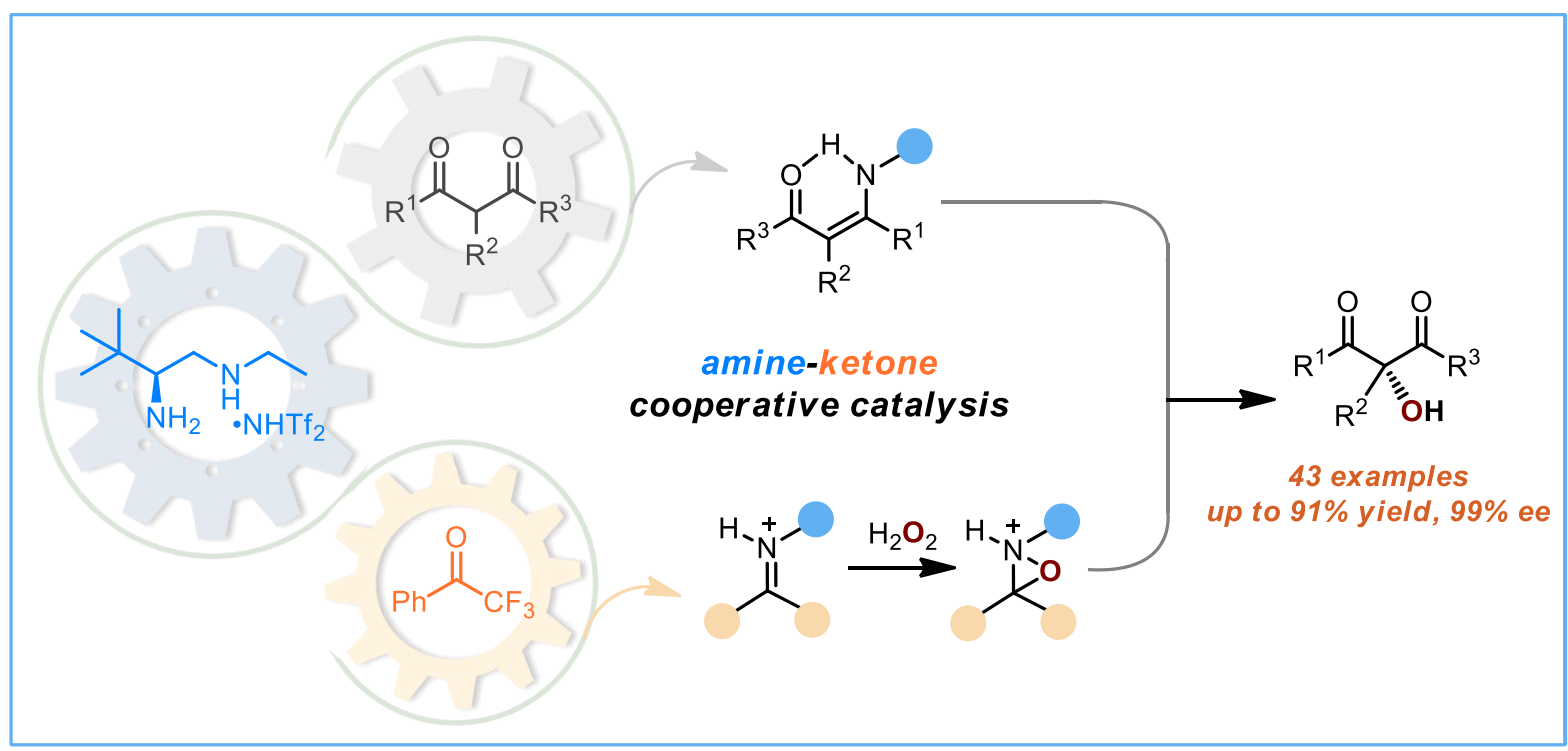

